\title{
El tiempo del diluvio. Interpretaciones evangélicas sobre el tiempo antiguo en el ayllu Coipasi, Bolivia
}

\author{
Óscar MuÑoz MorÁN \\ Depto. de Historia de América II (Antropología de América) \\ Universidad Complutense de Madrid \\ oscarmmoran@gmail.com
}

Recibido: 25 de mayo de 2013

Aceptado: 12 de septiembre de 2013

\section{RESUMEN}

En este artículo se utiliza la interpretación que los evangélicos hacen del tiempo más antiguo conocido en una comunidad quechua de Potosí, Bolivia, para plantear una doble discusión. En primero lugar, la propia capacidad de los evangélicos para crear y reproducir un discurso sobre el pasado que no entre en contradicción con el conocimiento tradicional del mismo. Por otro lado, interesa especialmente presentar la adaptabilidad a la contemporaneidad del sistema de creencias indígenas, es decir, analizar las diferentes representaciones del pasado que surgen al amparo de los cambios constantes a los que están sometidas las comunidades indígenas.

Palabras clave: Quechuas, Bolivia, sistemas de creencias, evangélicos, tiempo antiguo.

\section{The Time of the Flood. Evangelical Interpretations of the Ancient Time in the Coipasi Ayllu, Bolivia}

\begin{abstract}
In this paper I use the Quechua evangelical interpretation of the ancient time, to show a couple discussion. First, I ask how the evangelicals create and reproduce a speech about the past and it does not conflict with traditional knowledge. On the other hand, I want to present the adaptability to the contemporaneity of the system of indigenous beliefs, that it to say, I would like to analyze the different representations of the past that arise under the cover of constant changes that indigenous communities are subjected.
\end{abstract}

Key words: Quechuas, Bolivia, systems of beliefs, evangelicals, ancient time.

Sumario: 1. Introducción. 2. El conocimiento tradicional sobre el «tiempo del diluvio». 3. El «tiempo del diluvio» en la interpretación evangélica. 4. El «tiempo del diluvio» según el primer ministro evangélico. 5. Conclusiones. 6. Referencias bibliográficas.

\section{Introducción}

La realidad de la presencia del protestantismo en las comunidades amerindias es tan evidente que el análisis del discurso y las prácticas sociales que sus miembros ponen en circulación ocupa cada vez más espacio en las etnografías contemporáneas. Los protestantes son hoy en día cerca del 10\% de la población americana (Bastian 2006: 47; Laporta 2005: 305) y, en algunas regiones indígenas, muy por encima de esta cifra $^{1}$.

1 Por ejemplo para a comienzos de este siglo (y parece evidente que han ido en aumento desde entonces), El Salvador o Haití tenían por encima del 12\%, Brasil el 16\%, Guatemala el 19\% y los países con mayor población protestante eran Puerto Rico con el 20\% y Chile con el 21\%. Bolivia estaba alrededor del 7\% (Stoll en Marzal 2002: 418). 
Los amerindios protestantes, son parte de esa continua transformación a la que esta población se encuentra sometida; uno más de los múltiples campos donde poder apreciar el cambio social, así como su lugar en la modernidad. El resultado es la creación de nuevos códigos de significación, entre ellos, el del orden social, los aspectos rituales o los procesos cognitivos por medio de los cuales se encuentra sentido al tiempo y al espacio.

La literatura etnográfica sobre la presencia evangélica en las comunidades amerindias, se ha centrado, hasta el momento, en destacar la conflictividad que supone la ruptura por parte de los conversos de las normas sociales y los valores del grupo, así como de las transformaciones que esto ha provocado a los sistemas tradicionales ${ }^{2}$. En los Andes, el ámbito territorial que aquí nos ocupa, se ha hablado hasta la saciedad de su enfrentamiento con los procesos rituales y su negación del corpus de creencias panandinas.

«La identidad del grupo religioso [pentecostal] se construye a partir de una ruptura radical con el 'mundo', ruptura que pasa por el rechazo y el abandono de las 'costumbres', de las prácticas y 'supersticiones' de los no convertidos, llamados 'católicos' o 'paganos'» (Rivière 2007: 6).

Pocos han sido los que se han adentrado en el análisis del sistema de creencias propio de los evangélicos. Manuel Marzal ha llegado a decir que el discurso de estos transmite «un mensaje popular, sencillo y lleno de ejemplos, rico en citas bíblicas y pobre en reflexiones teológicas» (Marzal 2002: 510 y 514). Incluso Héctor Laporta en su reflexión sobre la religión evangélica en los Andes afirmó que las ideas de ésta son simplemente «experiencias y prácticas carismáticas, es decir, sin control de la razón» (Laporta 2005: 304).

También Manuel Marzal (Marzal 2002: 503) ha mencionado que las reflexiones de los protestantes americanos, y más concretamente de la población indígena, han provocado que se den en este continente nuevas formas de representación de la realidad donde la supuesta ruptura con la tradición ${ }^{3}$, aunque cierta, está sujeta a múltiples matices y estructuras culturas que nos llevan a afirmar que existen verdaderos intentos por parte de los evangélicos de conservar lazos con su sistema de creencias tradicionales ${ }^{4}$.

En este trabajo me interesa explorar uno de estos caminos: el de la lectura evangélica amerindia del pasado comunitario. O, dicho de otro modo, la forma cómo los evangélicos construyen una narrativa sobre el pasado de una comunidad quechua,

2 Al respecto quiero destacar los trabajos de los años sesenta del siglo pasado de Lalive d'Epinay (1968) en Chile o el de Juliana Strobele entre los aymaras del altiplano boliviano dos décadas después (1989). Más recientemente los de Susana Andrade en Chimborazo, Ecuador (2004 y 2005) y para la región que aquí nos ocupa, Bolivia, el de Giller Rivière (2007).

3 Es destacable el ejemplo de la Asociación Evangélica de la Misión Israelita del Nuevo Pacto Universal del pastor Ezequiel Alaucusi Gamonal, creada en el Perú en 1957 y cuyo poder teológico se basa en insertar los Evangelios dentro de la historia peruana conocida (Torre López 2005).

4 Estamos hablando aquí del concepto de «latinoamericanización del pentecostalismo» acuñado por J.P. Bastian: «en lugar de seguir la hipótesis de David Stoll (1990) o incluso de David Martin (1990) según la cual América Latina se estaría volviendo protestante hoy en día, al contrario, pienso yo que los protestantismos (...) se han latinoamericanizado con el pentecostalismo, al punto de asimilarse a la cultura religiosa de la región» (Bastian 2006: 53). Retomaremos en las conclusiones el debate sobre esta propuesta. 
Coipasi, cercana a Potosí, sin romper con el conocimiento comunitario y sin entrar en contradicción con lo que indica el Génesis. Es, al fin y al cabo, rastrear los caminos comunes que los evangélicos encuentran entre el mito local y las enseñanzas de la Biblia.

Tema que, por otra parte, ya ha sido tratado por parte de la literatura etnográfica que se ha ocupado de las iglesias evangélicas. Aunque, y aquí es donde creo que está la diferencia con este texto, siempre se ha puesto la atención en un elemento conocido y resaltable del argumentario protestante: la profecía o mesianismo de la llegada de Cristo y la destrucción de la actual corrupta humanidad (entre otros Wachtel 2001: 606). Como resume Marzal:

«hay que tener en presente, por una parte, que las raíces autóctonas pueden ser más bien un caldo de cultivo para el proselitismo evangélico. Por ejemplo, la 'escatología' del mundo cíclico y el 'mesianismo' del mito de Inkarrí, tan arraigados en la cultura andina, pueden hacer más atractivo el discurso pentecostal sobre el milenio y el discurso sobre el fin cercano de las confesiones escatológicas» (Marzal 2002: 493) .

Pero muy pocos se han centrado en la interpretación evangélica del pasado y, más concretamente, del tiempo más antiguo, el chullpa timpu ${ }^{6}$. Encontramos menciones a la relación conflictiva de los creyentes con los ancestros y antepasados, entre ellos, los chullpas o habitantes del tiempo antiguo. Rivière menciona que es común atribuir «a los pentecostales el saqueo de los santuarios y sitios prehispánicos-asociados a los fundadores ancestrales de la comunidad-y de las chullpas (...), cuyas momias son dispersas sin temor alguno a las consecuencias» (Rivière 2007: 7). Solo Wachtel describe un episodio parecido, cuando nos narra que en Chipaya los católicos o «paganos» solicitaron a los «hermanos» que destruyeran un mallku considerado especialmente maligno y que estos lo hicieron «con mucho placer» (Wachtel 2001: 599 y 616$)^{7}$.

Resulta sorprendente este vacío, ya que estamos ante un ejercicio interpretativo siempre imaginativo y abierto por parte de los evangélicos, que en su mayoría han crecido siendo parte del conocimiento tradicional de este tiempo antiguo y ahora tienen que encontrarle sentido dentro de una de las partes más importantes de la Biblia para su iglesia, el Génesis. Es probable que sean ciertas las acusaciones a la Iglesia

\footnotetext{
5 Más o menos en la misma línea nos dice Bastian: «La asimilación entonces de estos protestantismos por la cultura religiosa y política de los milenarismos y los mesianismos, permite que los percibamos hoy día más bien en continuidad que en ruptura con el universo cultural y religioso latinoamericano» (Bastian 2006: 51).

6 Existen algunos ejemplos en tierras bajas, como el estudio de Florencia Tola sobre los toba del Chaco argentino. En él, la autora realiza un análisis sobre las estructuras comunes (prácticamente universales) entre la interpretación evangélica toba o qom de su pasado mítico y los episodios recogidos en el Génesis (Tola 2011). Nuñez del Prado nos brindó en 1970 un relato maravilloso sobre el tiempo antiguo en la comunidad cuzqueña de Qotobamba. En él, aunque en ningún momento se menciona que sea de autoría evangélica, aparece la narrativa sobre los machus enriquecida con elementos bíblicos. Por ejemplo, los ñaupa-machus que habitaban entonces la tierra quisieron acabar con Cristo guiados por algunos de ellos que se llamaban Herodes, Pilatos y Caifás. De igual forma, tras la destrucción de éstos por un diluvio de fuego, creo Dios una nueva humanidad a partir de la pareja Arran e Iwa (tal vez Adán y Eva) que tras comer una manzana del arbusto prohibido convirtieron a todos sus descendientes en pecadores (Nuñez del Prado 1970: 65-67).

7 Cerca del lago Titica los altares ceremoniales del cerro Pachjiri fueron también cubiertos por los pentecostales con pinturas que indicaban «sus proclamas, en un intento de reconquista simbólica de los lugares sagrados dedicados a los rituales tradicionales» (Fernández y Albó 2008: 251).
} 
Evangélica respecto a su «fácil teología» (Marzal 2002: 514). También es probable que esto sea más claro dentro de las instituciones o los sectores que establecen las bases de dicha iglesia, pero creo que la insularidad de las comunidades indígenas americanas y su alejamiento de estas instituciones eclesiásticas, les hace ser más abiertas y libres para aceptar otras formas de interpretación de las Sagradas Escrituras ${ }^{8}$. Arturo de la Torre, que curiosamente analiza en su artículo cómo la Asociación Evangélica de la Misión Israelita del Nuevo Pacto Universal lee el Génesis en su relación con la historia del Perú, lo mencionaba muy bien respecto a la lectura que los grupos evangélicos hacen de la Biblia Reina-Valera: «la hilación [sic] de las enseñanzas resulta extremadamente abierta y de gran riqueza de posibilidades» (Torre López 2005: 326).

Veamos, por tanto, como en una pequeña comunidad quechua cercana a la ciudad de Potosí, los hermanos construyen un discurso propio sobre lo que sucedió en el lugar en el tiempo antiguo, sin abandonar por completo el conocimiento tradicional ${ }^{9}$.

Coipasi es el nombre de una comunidad, pero también de un ayllu y de un cantón, pertenecientes a la municipalidad de Chaquí, cercana a la ciudad de Potosí, en Bolivia. Coipasi es la cabeza del ayllu del mismo nombre, el cual, a su vez, está dividido en Ura Coipasi y Pata Coipasi. La comunidad de Coipasi pertenece al primero de ellos. Aunque la población censada esta entorno a los 1000 habitantes, lo cierto es que tan sólo aproximadamente la mitad vive en la comunidad, pues el resto son emigrantes de larga duración (principalmente en Argentina, aunque también en Santa Cruz o ciudades cercanas como Sucre o Potosí) e incluso permanentes.

\section{El conocimiento tradicional sobre el «tiempo del diluvio»}

Parece ser un lugar común en la Antropología de hoy que los grupos indígenas manejan (como sucede con el resto de estructuras culturales) una serie de códigos propios para dotar de significado e interpretar su pasado. También parece cada vez más compartido que esos códigos no son estáticos y que tienen una capacidad realmente asombrosa para adaptarse a la modernidad, con sus novedosas formas de conocimiento y su aluvión de información y contenido. Es decir, además del sistema propio (el que comúnmente denominamos tradicional) de interpretación del pasado, los indígenas reciben cada vez más información que complementa éste: datos históricos, técnicas e interpretaciones arqueológicas, lecturas incentivadas por los planes turísticos, aportes desde la paleontología o la misma antropología. Además aparecen nuevas formas de lectura del pasado desde la escuela, los movimientos políticos o las religiones emergentes.

La lectura comunitaria del pasado surge entonces confusa ante el etnógrafo. Se mezclan discursos donde lo que reconocemos como tradicional incorpora elementos

\footnotetext{
8 Tengo mis dudas, por tanto, que la frase de Manuel Marzal de que «bajo la fácil teología se esconde el miedo a la libertad; parece creerse en la uniformidad más que en la unidad» (Marzal 2002: 514), se pueda aplicar a lo que sucede en gran parte de las comunidades indígenas americanas.

9 Los evangélicos de Coipasi prefieren referirse a ellos mismos como «hermanos» o «creyentes», en ningún caso como evangélicos. En todo caso, el resto de la comunidad a veces los mencionan como «evangelistas». En este trabajo se utilizaran indistintamente unos y otros para hacer referencia a un mismo colectivo.
} 
de la modernidad. Los mitos que conocemos de las etnografías clásicas van dando paso a narraciones contemporáneas donde teniendo algo de aquellos son, sobre todo, nuevas lecturas. Porque el pensamiento indígena no es estático ni tradicional. Aunque tampoco moderno. Es simplemente contemporáneo, es decir, algo que hoy en día funciona con códigos del presente para crear un discurso presente.

Existe un esfuerzo comunitario por mostrar un conocimiento común del pasado, pese a la gran variedad de discursos sobre el mismo que nos podemos encontrar en la comunidad. Como ha mostrado Andrew Canessa en la comunidad aymara de Wila Kjarka, varían entre generaciones y entre géneros (Canessa 2012: 87), pero también entre aquellos que han sido autoridad y los que no, entre los que han emigrado o han permanecido siempre en la comunidad. Y, por supuesto, también entre los diferentes grupos religiosos.

En Coipasi el conocimiento sobre el unay timpu o chullpa timpu que podemos identificar para el resto de los Andes (principalmente sur del Perú y Bolivia) se presenta bajo unos parámetros diferentes a lo que comúnmente se ha registrado en otros lugares. Recordemos que, aunque con algunas variaciones, en el resto de la región se habla de una primera época en la que la tierra estaba habitada por unos seres claramente diferentes a los hombres (runas) actuales.

Estos seres (conocidos mayormente como machus en el Perú, chullpas o gentiles tanto en éste país como en Bolivia ${ }^{10}$ ) vivían sin la luz del sol, en algunos casos en completa oscuridad, en otros muchos en una noche perpetua, iluminada en cierta forma por la presencia de la luna y de las estrellas. Los chullpas se presuponen que vivían en un estado semisalvaje, sin poder cultivar nada, solo les quedaba alimentarse de piedras y sin sal. La mayoría de los relatos no se extienden más en detalles sobre la cotidianidad de los chullpas, pues el apocalipsis que dio lugar a su desaparición se ha convertido en el cuerpo central de las narraciones. Sí coinciden, no obstante, en que vivían en pecado, es decir, fue una humanidad fallida. Conscientes de este hecho temían la llegada de Dios y, en algunas narraciones, lo persiguen por la tierra para destruirlo (p. ej. Rasnake 1988).

Tras enterarse los chullpas de la llegada de Dios a la tierra, decidieron esconderse algunos en cuevas u oquedades, lugares liminales que hoy se presuponen conectan diferentes estados y espacios ontológicos principalmente en las qaqas (Cruz 2006), aunque la mayoría se construyó casas con una única puerta orientada hacia el naciente. Allí aguardaron escondidos la llegada de Dios, el cual hizo su aparición en forma de sol ${ }^{11}$. Cuando éste salió arrasó con toda la vida en la tierra, incluida la de los chullpas que fueron quemados y secados al pasar los rayos por las puertas de las viviendas

10 La bibliografía sobre los(las) chullpas en los Andes es muy abundante (Sendón 2010) y por eso quiero señalar aquí solo algunos ejemplos al respecto. En el Perú, donde predomina el término machu, cabe destacar el trabajo de Catherine Allen (1994) y de Ricard Lanata (2007), así como el ya clásico de Manuel Marzal sobre Urcos (1971). En el altiplano boliviano los de Thomas Abercombrie (1998) o Roger Rasnake (1988). $\mathrm{Y}$, por último, en la región lacustre de los uros, conocidos como los descendientes directos de los chullpas, los trabajos de Nathan Wachtel (2001) y el pionero, pues registró tal vez por primera vez el mito, de Alfred Métraux (1931).

11 En algunos relatos, los menos (p. ej. Casaverde 1970: 151), Dios envía un diluvio de fuego en vez de aparecer él en forma de sol. En el caso de Coipasi que veremos a continuación, se habla siempre de diluvio de fuego. 
construidas. Solo lograron salvarse aquellos que se habían escondido en cuevas, por medio de las cuales accedieron al interior de la tierra para, posteriormente y según las versiones registradas en las comunidades lacustres del altiplano boliviano (Métraux 1931 y Wachtel 2001), aparecer de nuevo en los lagos y dar origen a lo que hoy en día son los uro-chipaya ${ }^{12}$.

En la comunidad de Coipasi hay chullpas, aunque claramente éstas se refieren a los restos que hoy se pueden encontrar de esta civilización antigua ${ }^{13}$. Los seres que formaban esa civilización son denominados en términos generales «gentes» y convivían con animales (salvajes todos ellos, algunos los actuales, otros ya extintos). En ningún momento se menciona que vivieran en la oscuridad o tuvieran un estilo de vida diferente al actual. Más bien todo lo contrario, «eran como nosotros» y vivían en un pueblo, Laqaya ${ }^{14}$, donde tenían su plaza y su iglesia incluida. Todos coinciden, no obstante, en que estamos hablando en un tiempo anterior a Cristo y que, eso sí, deberían de vivir en una especie de pecado colectivo porque Dios les castigó en forma de diluvio de fuego que acabó prácticamente con todos ellos. Solo algunos consiguieron protegerse en una cueva cercana (Vizcacha Wasi) desde donde fueron haciendo incursiones al interior del cerro Phutina, donde está situada. Allí se dice que llegaron a tener grandes salones y cocinas. Pero no se sabe qué fue de estos supervivientes ni se da una solución de continuidad en la narración, por tanto, a esta población («generación» le llaman algunos, especialmente los evangélicos ${ }^{15}$ ) se la da por desaparecida $\mathrm{y}$, por supuesto, los coipaseños no se identifican con ellos.

Este parece ser el conocimiento más tradicional. El narrado por las personas de más edad o por aquellos que han estado menos expuestos a aportaciones externas (los que apenas fueron a la escuela, no han emigrado nunca o tenido contacto con nuevas religiones). Otro caso diferente son aquellos que sí están sujetos a estas instituciones que han ido poco a poco incorporando referentes diferentes al relato tradicional.

Las escuelas comunitarias pertenecen a la red de Escuelas de Cristo que ya en la década de años veinte del siglo pasado estaban implantadas en gran parte del Departamento de Potosí (Choque y Quisbert 2006), aunque a Coipasi no llegaron hasta finales de la década de los cuarenta. Aquellos que comenzaron a ir a la escuela tras la

12 En las comunidades cercanas a las yungas de la selva, son los pueblos indígenas de ésta, conocidos habitualmente como chunchus, a los que se consideran los descendientes de los chullpas, como muy bien se refleja en los danzantes que los representan hoy (Molinié 2005 y Ricard 2007: 265).

13 En Coipasi los chullpas, en masculino, se usa en las pocas ocasiones en que se refieren con ese nombre a los habitantes de ese tiempo. Es más común escuchar el femenino las chullpas, y entonces se hace referencia a los restos óseos principalmente, pero también a las piezas de cerámica o cualquier otro objeto que se encuentre en zona chullpa y se adjudique a los que allí antes vivían.

14 No es fácil precisar la traducción de dicho término que por lo general está ausente de los diccionarios de quechua. Algún coipaseño me afirmaba que quiere decir «así como solares, sin casas». En el diccionario AULEX online Quechua-Español aparece la traducción de «Casa en ruinas», lo cual concuerda perfectamente con el referente que los coipaseños tienen del lugar. Francisco M. Gil también registró en Lípez este topónimo para referirse al pueblo de los antiguos, y en su caso lo traduce como «pueblo en ruinas» (Gil 2007: 664).

15 Dice Valeriano Rodríguez: «Porque esa ha sido la primera generación que ha pasado [la de Laqaya]. Ahora está una segunda generación [la actual]. Ahora digamos el castigo es con fuego y azufre [el que vendrá para crear una nueva generación solo de creyentes]». Este discurso, al igual que las profecías del Inkarrí, es bien conocido en la mitología andina, pues en muchas ocasiones se habla de las tres generaciones: la del Padre que en términos generales se refiere al chullpa timpu; la de Jesucristo o el Hijo, que es la actual; y la del Espíritu Santo que está por venir (p. ej. Marzal 1971 y Rasnake 1988). 
Revolución de 1952, recibieron de ésta una enseñanza en profundidad de la Biblia, provocando la transformación en los relatos en gran parte de los comunarios.

Para estos, Laqaya y sus habitantes existían pero no fueron destruidos por un diluvio de fuego, sino por el bíblico de agua de 40 días y 40 noches. El agua inundó todo, hasta la cima de los cerros, acabando con todo tipo de vida, incluidos los animales. En muchos casos este diluvio universal arrastró animales de otros lugares y por eso en la pampa se pueden encontrar hoy restos de dinosaurios. Por ejemplo, don Luciano Gaspar me explica al respecto:

«Claro, con el diluvio. Les enterraron, dice. Claro cubrió el agua, cubrió todo, todo, todo. Claro, entonces, donde han asentado...eso animales no habrán existido aquí pues. No ves. No habrán existido. Habrán existido en otros países. En Oriente por ahí... algo. Con el movimiento del agua, ¿a dónde no han podido llegar? ¿no ves? Yo digo eso».

Los que mantienen esta teoría no consiguen explicar bien la existencia de sobrevivientes en el Vizcacha Wasi (de cuya existencia no se duda), y a las preguntas insistentes del antropólogo la sortean afirmando que como la cueva estaba más alta, allí pudieron refugiarse y también introduciéndose en el interior del cerro.

Los habitantes de Santiago K, en Lípez, afirman que existieron dos humanidades en esos tiempos antiguos: una primera de la que apenas se conoce nada y que pereció con el diluvio de agua, y una segunda, la de los chullpas, que vivían en la oscuridad y murió tras la salida del sol (Gil 2007: 664). En Coipasi en cambio, ambos diluvios afectaron aparentemente a la misma humanidad. Según las versiones, se habla incluso de un diluvio de fuego en Laqaya previo al de agua. Los dos son universales, pero el segundo parece tener un carácter más apocalíptico ya que acabó con el resto de seres del planeta, principalmente los animales.

Sea de una forma u otra, los coipaseños hablan de un diluvio como castigo divino y es por esta razón por la que el tiempo es conocido, de una forma bastante generalizada en toda la comunidad, como el «tiempo del diluvio».

\section{El «tiempo del diluvio» en la interpretación evangélica}

Los creyentes coipaseños (parte de la mayoría pentecostal en el país) son hoy una minoría, pues apenas representan el $10 \%$ de los residentes en la comunidad. Los comunarios afirman que el primer pastor evangélico y las primeras conversiones aparecieron en los años cincuenta del siglo pasado, aunque tan sólo una de las familias actuales de hermanos remonta sus conversiones a esa época. Se podría decir que el resto de ellos son nuevos, la mayoría convertidos durante sus periodos de emigración en tierras bajas. Lo habitual es que los llamados «viejitos» de la Iglesia tengan entre los sesenta y los setenta años y que estos fueran los primeros en convertirse estando en Santa Cruz o en Argentina (en los años setenta y ochenta del siglo pasado). Estos mayores son los que han ido evangelizando al resto de los comunarios, captando a sus familiares en primer lugar y, posteriormente, a personas afines (Stoll en Marzal 2002: 490). En los años noventa y primera década del siglo XXI, el número de creyentes en la comunidad, según todos los informantes, era mucho mayor, entre el 30 y el $40 \%$, pero como dicen ellos, «se han ido cayendo». Ahora, los evangélicos son entre vein- 
ticinco y treinta personas. No obstante, y pese a este marginal número, su presencia es de suma importancia en Coipasi.

En primer lugar porque esos tiempos pasados en los que el número de creyentes era mucho mayor, provocaba que casi todas las familias de la comunidad tuvieran entre sus miembros a un evangélico. La consecuencia es que sus discursos y prácticas sean bien conocidas por todos los coipaseños.

En la misma línea, los valores argumentados por los evangélicos son tan rompedores y agresivos respecto a la práctica tradicional que sin duda alguna no pasan desapercibidos para el resto de la comunidad ${ }^{16}$. Se convive con ellos, se tienen que salvar y contrarrestar ${ }^{17}$, se les culpa de muchos de los cambios que sufre el núcleo pero, y eso es lo realmente sorprendente e interesante, en muchos casos se interioriza y usa su discurso, al menos hacía el exterior de la comunidad (Muñoz s.f.). Sucede así, por ejemplo, con el uso excesivo del alcohol en las celebraciones tradicionales y contra el cual se muestran abiertamente hostiles los evangélicos. Este discurso, aunque seguro no tan virulento, puede escucharse incluso entre los católicos, al menos cuando reciben la visita de agentes externos como antropólogos, profesores de universidad, pero también alcaldes o miembro del gobierno del Departamento de Potosí.

De igual forma, pese a la tensión entre la práctica tradicional y la evangélica, el acceso a los cargos comunitarios no está vedado a los hermanos. Lo cierto es que participan de ellos como cualquier otro comunario, como sucedió durante mi estancia en 2011, año en el que las tres autoridades (kuraka, alcalde y corregidor) eran evangélicos ${ }^{18}$. Como autoridades, su palabra tiene más trascendencia y difusión y sus comportamientos alcanzan una esfera -la de la representación y dirección de los asuntos comunarios- que supera la individual y social. El kuraka durante 2011 era, además, el ministro principal de la iglesia evangélica comunaria y uno de los principales informantes de este trabajo.

El día 1 de noviembre, primera fecha de las dos de celebración de la fiesta de Todos los Santos, me encontraba en la casa de don Vicente donde se estaba velando a su mujer doña Angélica. Ésta era evangélica, aunque no su marido. No obstante, éste había decidido hacerle - tal vez, en un ejemplo más de convivencia de los dos cultosun acto bajo los preceptos de esta iglesia. En él se encontraban los tres principales ministros o ancianos evangélicos, a la cabeza de ellos el kuraka. Durante el almuerzo en el patio de la casa, estaba dialogando con éste y con don Luis Huaranca, un coipa-

\footnotetext{
$16 \mathrm{Al}$ respecto, Andrew Canessa ha planteado recientemente una interesante nueva perspectiva que puede explicar gran parte de los discursos anti-tradición que hemos escuchado a los evangelistas de las comunidades que estudiamos. Según Canessa la conversión y el discurso evangélico en Bolivia, tiene mucho que ver con una condición «racial» y «jerárquica», un deseo de avanzar desde lo indio (jaqi) hasta lo occidental, sin pasar por lo mestizo ( $q^{\prime}$ ara) (Canessa 2000 y 2012).

17 Nos dice Marzal que los cuatro principales caminos de «resistencia de los católicos son: el compadrazgo e importancia de los momentos claves del ciclo vital»; «la fiesta patronal, que es la experiencia religiosa de los pueblos»; el catequismo familiar y social; y la devoción mariana (Marzal 2002: 492).

18 El kuraka y el corregidor son los dos elegidos a finales del mes de noviembre y toman posesión el día 6 de enero. El alcalde en cambio lo hace en el mes de junio. Durante el año 2011 el alcalde de la primera mitad del año era católico y claramente tuvo él que llevar gran parte del peso de las responsabilidades de la comunidad ante la apatía de las otras dos autoridades. En junio entro un nuevo alcalde, soltero, joven y evangelista (fue elegida, en realidad, su mamá y funcionaban como pareja de autoridades), que fue muy criticado por la comunidad por su falta de compromiso con el cargo.
} 
seño maestro y residente en la ciudad de Potosí. Don Luis me preguntaba por mi trabajo, pues no habíamos tenido la oportunidad de conocernos hasta ese momento. $\mathrm{Al}$ comentarle que era maestro de Historia en España, asegura que entonces debo saber dónde cayó el meteorito o «cuerpo celeste», refiriéndose a lo que sucedió en Laqaya y el Vizcacha Wasi. Me comenta que se puede comprobar que en los alrededores de este último lugar aparecen ennegrecidas muchas piedras (detalle que, reconozco, no había observado pese haber visitado el sitio en innumerables ocasiones. Pude comprobar al día siguiente que era cierto ${ }^{19}$ ). Don Luis menciona el relato tradicional según el cual «los hombres» que por allí vivían (se refiere a la cercana Laqaya) tuvieron que refugiarse del diluvio de fuego en la cueva cercana.

Tata kuraka Guillermo que nos estaba escuchando responde que sí sucedió eso, al igual que en Sodoma y Gomorra, que fueron castigados por Dios debido a sus innumerables pecados. Dios hizo caer sobre ellos un diluvio de fuego, reconociendo que desde pequeño él escuchaba a sus abuelos narrar como la gente de Laqaya fue castigada con un diluvio de fuego.

Porque para los evangélicos existieron los dos diluvios, de fuego y de agua, como bien refleja el Génesis. Aunque no parecen estar muy seguros de cual afectó antes a Laqaya, afirmando la mayoría que primero fue el de fuego y salvándose bastantes de los habitantes de Laqaya en el Vizcacha Wasi; y posteriormente, el de agua que acabó ya con todo, aunque hablan mayormente de los animales y no tanto de los hombres. Veámoslo con un poco más detalle.

La mayoría de los hermanos hemos comentado que se convirtieron durante su periodo de emigrantes. Los debemos ubicar, por tanto, entre la franja de edad que va de los cuarenta y cinco a los setenta años, es decir, son también aquellos escolarizados desde los años cincuenta e incluso un poco antes. Quiere esto decir que muchos de ellos comenzaron a incorporar a sus conocimientos sobre el pasado, la lectura bíblica que les proporcionaban los maestros de la Escuela de Cristo de la comunidad. Como ya he mencionado que afirman los coipaseños, parece ser que el diluvio de agua, como cataclismo que acabó con Laqaya, comenzó aparecer como sustituto del de fuego por estos años.

Así, los evangélicos mencionan que fue con el diluvio de los 40 días y las 40 noches cuando toda la tierra se inundó y Laqaya y sus gentes desaparecieron:«Entonces, antiguamente como era, ha pasado aquella vez un juicio. No sé si sabe, es cierto. Entonces el juicio ha pasado con diluvio, ¿no es cierto? El mundo se ha terminado con agua aquella vez, el primer juicio de Dios, que ha hecho, ¿no es cierto? Entonces aquel última roca, digamos, el monte alto, siete kilómetros, si no me equivoco... siete metros más ha pasado la lluvia. Ese diluvio. Entonces por esa razón todo el mundo se ha hundido con agua. O sea toda la gente se ha muerto con agua. El único que se ha salvado ha sido el Noé con su familia. Por obediente a Dios, de aquella vez. Entonces de ahí, pues, esa vez había también como ahora: el ser humano, animales, bestias.... todo había tenido aquí, ¿no es cierto? Entonces como se ha hundido, pues, todo se ha enterrado. De ahí aparecen los huesos. Se han quedado ahí animales que, gigantes

19 Casaverde Rojas también registró en el Cuzco que se usaba el mismo hecho (piedras ennegrecidas) para justificar la existencia del diluvio de fuego que acabó con los machus (Casaverde 1970: 152). 
que había...personas que había, es que han muerto, ese están apareciendo ahora, pues. Había... más antes dice que cocinaban en ollitas, vasitos también había... entonces... piedras también así pa moler, pa moler trigo, ají.... había pues. Esas cosas están apareciendo ahora. Entonces aquí en Coipasi hay mucho de eso» (Valeriano Rodriguez).

Los evangélicos, a diferencia del resto de la comunidad, incorporan a la narración sobre el pasado de Coipasi el pasaje bíblico del diluvio de agua con muchos más detalles:

«Dios se enojó y dijo 'Ya'. Y Noe, obediente 'hazte una arca porque yo he decidido enterrar este mundo'. Así se enojó Dios. Entonces ha hecho llover 40 días y 40 noches ha hecho llover Dios. Entonces en ese tiempo que ha llovido, tanta agua que se ha juntado, ha subido, ha subido, ha subido último cerro, el más alto de cerro, siete metros más de agua, de altura de agua, pues. Todito lo ha hundido pues. Tapó todo. Dice la Biblia» (Valeriano Rodriguez ${ }^{20}$ ).

Resumamos. Según los hermanos, Laqaya (no Coipasi, que está claro para sus habitantes que entonces todavía no existía) fue afectada por el diluvio como el resto de la tierra. De hecho, al preguntarle a doña Alberta Potosí por las chullpas, me dice: «Usted en allá debe saber también. En todas partes fue ese diluvio», refiriéndose que también en mi país debe haber restos de los antiguos. Además, como sostienen los comunarios, al ser un diluvio universal de agua, los restos de las personas y animales muertos (especialmente de éstos) se vieron arrastrados de unos lugares a otros.

En Laqaya, con el diluvio de agua, murieron todos sus habitantes, pero, remarcan los evangélicos, especialmente los animales que había en el lugar. Porque el nivel de las aguas subió tanto que alcanzó casi la cima de los cerros más altos que se pueden divisar desde Coipasi (en este caso, el Tumillki, de 4.035 metros de altura). Así lo menciona el Génesis:

«Y fue el diluvio cuarenta días sobre la tierra; y las aguas crecieron, y alzaron el arca, y se elevó sobre la tierra. Y subieron las aguas y crecieron en gran manera sobre la tierra; y flotaba el arca sobre la superficie de las aguas. Y las aguas subieron mucho sobre la tierra; y todos los montes altos que había debajo de todos los cielos, fueron cubiertos. Quince codos más alto subieron las aguas, después que fueron cubiertos los montes. Y murió toda carne que se mueve sobre la tierra, así de aves como de ganado $\mathrm{y}$ de bestias, $\mathrm{y}$ de todo reptil que se arrastra sobre la tierra, $\mathrm{y}$ todo hombre. Todo lo que tenía aliento de espíritu de vida en sus narices, todo lo que había en la tierra, murió» $\left(\right.$ Génesis 7,17) ${ }^{21}$.

20 Sorprendentemente un día fui a visitar a don Luciano Gaspar, uno de los tres ministros evangelistas, y se encontraba leyendo la Biblia en el patio de su casa. Nos sentamos a conversar y le pregunté por el pasaje del Génesis donde aparecía «el diluvio de Noé». Extrañado, Luciano ojeó la Biblia, pasando sus páginas hacía adelante y hacía atrás. A una pregunta mía confiesa que nunca lo había leído y tuve que indicarle yo dónde se encontraba. A continuación me lo leyó tal cual aparece en el Génesis: «Y he aquí que yo traigo un diluvio de aguas sobre la tierra, para destruir toda carne en que haya espíritu de vida debajo del cielo; todo lo que hay en la tierra morirá. Más estableceré mi pacto contigo, y entrarás en el arca tú, tus hijos, tu mujer, y las mujeres de tus hijos contigo. Y de todo lo que vive, de toda carne, dos de cada especie meterás en el arca, para que tengan vida contigo; macho y hembra serán. De las aves según su especie, y de las bestias según su especie, de todo reptil de la tierra según su especie, dos de cada especie entrarán contigo, para que tengan vida» (Génesis 6,17).

21 La versión de la Biblia que aquí se cita es la utilizada mayormente por las iglesias evangélicas: la denominada Reina-Valera (La Santa Biblia 2005). 
A raíz de lo que nos presenta este pasaje, los evangélicos coipaseños no parecen concederle muchas alternativas a los que vivían en Laqaya:

«Todito. Todo el mundo [ha muerto con el diluvio]. Después nosotros estamos pues. Antes había otra gente, igual que nosotros... había, había... dice que era gente muy pecadora» (Valeriano Rodríguez).

Algunos de los hermanos a los que les preguntaba por el diluvio de fuego negaban su existencia en Laqaya, aunque no en Sodoma y Gomorra. Eso sí, y aquí es el momento de referirnos a las famosas teorías mesiánicas evangélicas, al igual que Dios mandó el castigo a estas dos ciudades por pecadoras ${ }^{22}$, lo mandará próximamente a esta tierra habitada ahora, como entonces (se usa como comparación tanto a los habitantes de Sodoma y Gomorra, como las gentes de Laqaya), por una humanidad corrompida. Solo los creyentes se salvarán:

«Y en todos mis montes llamaré contra él la espada, dice Jehová el Señor; la espada de cada cual será contra su hermano. Y yo litigaré contra él con pestilencia y con sangre; y haré llover sobre él, sobre sus tropas y sobre los muchos pueblos que están con él, impetuosa lluvia, y piedras de granizo, fuego y azufre. Y seré engrandecido y santificado, y seré conocido ante los ojos de muchas naciones; y sabrán que yo soy Jehová» (Ezequiel 38.21).

Sí resulta algo más común en el resto de los Andes, encontrar referencias al pasado chullpa para explicar cómo sucederá la nueva extinción de la humanidad. Wachtel lo registró en lo que denominó la «patética» orientación del pentecostalismo chipaya hacía el «fin de los tiempos» (2001: 601). Le dice un informante:

«(...) Luego caerá una lluvia de fuego que durará siete años, purificará la tierra y consumirá la humanidad.

Sin embargo, no todos los hombres morirán; algunos sobrevivirán al fuego, como antiguamente los chullpas, una pareja de cada raza, un hombre y una mujer chipayas, un hombre y una mujer aymaras, un hombre y una mujer gringos, etc, que volverán a poblar la tierra» (Wachtel 2001: 606) ${ }^{23}$.

En un municipio cercano a Coipasi, Tinkipaya, Vincent Nicolas también nos presenta un relato donde se relaciona a los chullpas, su extinción y la que está por venir según los evangélicos. La diferencia en esta narración es que este nuevo cataclismo será un diluvio de agua:

«La gente chullpa de antes, en el fuego ha muerto, pues. El fuego le ha quemado cuando el Sol ha salido. Ya. Al lado donde sale el Sol habían hecho la puerta de sus casas. El Sol ha entrado dentro de la casa, ha matado a ese chullpa. El padre era ese chullpa $\mathrm{y}$, ahora, nosotros somos cristianos. Entonces, en el agua, vamos a morir nosotros. Ahí, ahora, cuarenta días va a llover dicen (...)» (Nicolas et al 2004: 25).

\footnotetext{
22 «Entonces Jehová hizo llover sobre Sodoma y sobre Gomorra azufre y fuego de parte de Jehová desde los cielos; y destruyó las ciudades, y toda aquella llanura, con todos los moradores de aquellas ciudades, y el fruto de la tierra» (Génesis 19,24).

23 El relato, bastante más extenso, continúa hablando del retorno al mundo de oscuridad y de agua como en la época de los chullpas.
} 


\section{El «tiempo del diluvio» según el primer ministro evangélico}

No todos los hermanos en Coipasi niegan la tradicional versión del diluvio de fuego como el que acabó con Laqaya. Tata Guillermo tiene otro relato diferente. Es importante recordar brevemente que Tata Guillermo es el primer anciano de la iglesia evangélica en la comunidad. Es claramente reconocido como el mayor estudioso y conocedor de la Biblia. Y, sin duda alguna, el que con más entusiasmo practica el proselitismo entre todo aquel que quiera escucharle. Conociendo la Biblia al detalle, utiliza constantemente sus mensajes para explicar muchos de los valores de la vida. Además, y lo que resulta más importante, ha construido un discurso sumamente elaborado y de gran fuerza y convicción para justificar cualquier tipo de práctica evangélica frente al desafío de la tradicional ${ }^{24}$. Su interpretación del pasado también ha seguido esta línea.

Tras el encuentro más o menos informal el día de Todos los Santos, acudí a conversar extensamente con él, pero en calidad de primer ministro de la iglesia evangélica, con el objetivo de que me explicara bien sus palabras de ese día. Yo tenía especial interés en ver porqué él, a diferencia del resto de los creyentes, seguía manteniendo que la lluvia que destruyó Laqaya era de fuego y no de agua.

«Sí, una lluvia de fuego. Por eso y entonces, mi papá me dijo, antes (...) bueno según la Biblia lo que enseña, la creación del universo no pasa más de... pasa más de 6000 años. Según esta Biblia que tenemos. Más de 6000 años. Ahora, desde la creación del universo, cuando Dios ha formado en 6 días, listo. En 7 días descanso. A veces trae pensativo, ¿no? Porque para Dios, dice, un día es como 1000 años. 1000 años. Ese 1000 años es como un día. Vayamos a ver, en 6 días creó....casi va a ser como 7000 años. Entonces y ahora (...) desde la creación del universo hasta el diluvio, entonces pasaron 2000 años. En esos 2000 años entos dice que multiplicaron mucha gente. Igualito dicen que en ese tiempo llenaban la tierra. Porque para el caso, ya estaba la gente acá, allá, aquí. En Sudamérica, en Centroamérica, en Asia, después Europa, en....cómo se llama, el Viejo Mundo, como se llama, para el lado de África. Entonces ya había gente ya. Entonces lo cual, dice que la gente vivía siempre en pecado, en pecado. Bueno, hoy día también lo mismo (...). Lo mismo vive la gente. Bueno. Dice, aquel, había dos, digamos, éste, dos pueblos grandes. De decir, Sodoma [y] Gomorra, ¿no? Eran una ciudad corrupta, una ciudad digamos, lleno de pecados...había dice ahí homosexuales, había afeminados, de todo había allí, de todas esas cosas (...) Como Dios había visto tanto ese pecado, esa corrupción, bueno, entonces había dicho, 'Lo voy a castigar, lo voy a castigar'. Entonces, 'lo voy a castigar con fuego, con fuego'. Entonces dice que Sodoma representa a todo el mundo, a todo el mundo. Y Gomorra es la ciudad, ese pueblito... Entonces, como dice don Luis, cayó un meteorito, ¿no? Dice él. Por eso, con relación a la Biblia tiene que ser siempre. Entonces, yo no creo, digo. Entonces... bueno entonces, dice que antiguamente la población existía ahí pues, ahí

24 Un ejemplo lo encontramos cuando se muestra sumamente crítico con prácticas comunitarias como el consumo de alcohol o coca, o el papel y representación de las autoridades tradicionales. Tata Guillermo aceptó el cargo en 2010 siempre dejando claro ante la comunidad que, a diferencia de otros evangelistas anteriores también nombrados kurakas, él nunca iba a renunciar a su condición actual, es decir, no iba a participar de celebraciones, fiestas o eventos ni como comunario ni como autoridad. Tampoco iba a portar los elementos distintivos de las autoridades tradicionales andinas: el crucifijo, la chuspa y el bastón de mando. Tata Guillermo fue fiel a su palabra, por lo cual fue fuertemente criticado dentro y fuera de la comunidad. 
existía [en Laqaya]. Claro que, seguramente, como éste, como antiguamente... ahora según estoy analizando también yo... entonces dice que después de la creación de todos los animales, seguramente, para Dios, como vivía la gente (...)».

Este es un extracto de una larga explicación en la que don Guillermo comienza haciendo clara referencia al conocimiento tradicional, «mi papá me dijo, antes», y reconociendo que entonces su papá y su abuelito sabían que fue un diluvio de fuego lo que destruyó Laqaya. Más adelante, en la misma conversación, aclara que

«Mi abuelo me contaba esto. Se llamaba Olegario Huaranca (...) Un día, pues fuimos, tenemos una huerta allá en la orilla [cerca de Laqaya], entonces (...) dice, 'mira hijito te voy a contar'. Dice 'que cuando, ¿ves aquella negra?', me dijo. Digo 'si'. 'Antiguamente, mi papá me contaba (dice) que caía fuego del cielo, (dice). Una lluvia de fuego caía, (dice). Entonces así me contaba mi abuelo. Entonces ahí siempre, digamos, así asito, negrito, hay unos dibujos, tipo de llamitas'. Entonces, ahora y esa vuelta claro, yo pienso, no, antiguamente como caía fuego del cielo, y seguramente como Gomorra, como Sodoma representa al mundo entero, pasó así acá también. Entonces como representa al mundo entero».

En su discurso, don Guillermo hace referencia a la creación de la tierra y la población de la misma. Entonces, dos ciudades se poblaron por encima del resto y se convirtieron en nidos de la corrupción y del pecado. Dios decide castigarles con fuego, el meteorito que don Luis Huaranca mencionó, y éste acabó con «la población [que antiguamente] existía ahí», en Laqaya.

Como menciona claramente en su narración, don Guillermo tiene como objetivo mantener una explicación e interpretación de los acontecimientos «con relación a la Biblia tiene que ser siempre». Aunque nunca abandonando el conocimiento tradicional, el aportado por sus abuelos y padres, la incorporación de la narrativa bíblica resulta imprescindible para entender, en el presente, qué sucedió en el pasado: «según estoy analizando también yo».

Posteriormente en la conversación insisto en preguntarle si él cree que Laqaya desapareció con el diluvio de fuego, a lo que me contesta en un par de ocasiones afirmativamente, que acabó principalmente con las personas que allí vivían. Los animales, en cambio, se extinguen en su mayoría con el posterior diluvio de agua:

«Óscar: O sea que usted piensa que Laqaya desapareció con un diluvio de fuego.

Guillermo: Diluvio de fuego.

Óscar: Que fue el diluvio de Sodoma...

Guillermo: Sí, de Sodoma y Gomorra. Porque me contaron mis abuelos. Me contaron mi abuelo así. Entonces cayó fuego ya. Entonces estos fósiles están pues, como se llama, del diluvio...

Óscar: ¿Pero del de fuego o del de agua?

Guillermo: Es del fuego, yo digo. Allá en Laqaya.

Óscar: ¿Y los de los animales...?

Guillermo: Los de los animales es del diluvio.

Óscar: Del de agua. Del de 40 días y 40 noches.

Guillermo: Del de 40 días y 40 noches. Porque dice, el diluvio arrasaba todo el mundo. No se veía ni cerros, ni nada. El pico más alto (...), hasta que tape ese cerro dice que no se veían picos más altos, dice que no se veían. 40 días y 40 noches son muchos días, 
80 días. Y como no se va a volver, como agua se habrá vuelto, qué se yo en la tierra. Seguramente al rato se abrieron grietas y enterraron pues a los animales ahí. De ahí son esas $\left.\operatorname{cosas}^{25}\right\rangle$.

Los habitantes de Laqaya murieron todos con el diluvio de fuego, aunque algunos consiguieron refugiarse en el Vizcacha Wasi, incluso haciéndose unos gorros de cuero para protegerse la cabeza:

«Cayó fuego. Cuentan, no. Pero me cuentan a mí también, cayó fuego. Y esa gente por no hacerse quemar, dice que entonces, por no sufrir con fuego, dice que escaparon ahí. Entonces se habían hecho, de gorro se habían hecho, con unos cueros se habían hecho. Entonces, que se ocultan, que sé yo. Y ahí tienen unas pinturas en forma de, ¿cómo se llama? de animalitos, de gentes. Ahí pues» (Guillermo Huaranca).

Resumamos entonces el tiempo del diluvio contado por Tata Guillermo. Hace muchos años, antes de Cristo, vivían en Laqaya unas «gentes» corruptas y pecadoras, el mismo comportamiento del que hacía gala el resto de la humanidad, aunque era especialmente significativo en Sodoma y Gomorra. Como Dios estaba muy enfadado con estas dos ciudades envió una lluvia de fuego para destruirlos, al igual en Laqaya (el meteorito mencionado por don Luis Huaranca). Esto se puede comprobar en el color negro que presentan las piedras cercanas al Vizcacha Wasi, algo puesto en valor por el conocimiento de los ancianos, como el de su abuelito. Todos los hombres fueron entonces destruidos, aunque al menos en Laqaya parece ser que algunos consiguieron salvarse cubriendo sus cabezas con cuero (dato que, por otra parte, solo escuché en esta ocasión) y escondiéndose en el Vizcacha Wasi. Posteriormente Dios, todavía molesto con el comportamiento de hombres y animales (no termina de quedar claro qué hombres son. Si son los supervivientes del primer diluvio de fuego u otra generación), decide enviar un nuevo diluvio, esta vez de agua, de 40 días y 40 noches. Este acaba con los seres vivos de la tierra, pero se pone el acento especialmente en los animales, consecuencia de lo cual podemos encontrar sus restos «fósiles» hoy en la pampa coipaseña.

Con estos dos castigos Dios acaba con todos los seres de la tierra, incluidos los hombres de Laqaya y los animales de la pampa. Ellos, los coipaseños actuales, son otra generación, diferente a aquella, aunque igual de pecadores. Por esta razón Dios enviará un nuevo diluvio de fuego y azufre para acabar con ellos. Solo se salvarán los evangélicos, los creyentes ${ }^{26}$.

25 Estas «cosas» son los huesos de dinosaurios que se encuentran en la pampa coipaseña y que antes ha denominado «fósiles». No tenemos espacio aquí para tratar este aspecto que se ha ido incorporando poco a poco a la narrativa local sobre el pasado más antiguo. Tan sólo mencionar que parece ser que hasta hace un par de décadas estos huesos eran atribuidos de una forma mayoritaria a chullpas de tamaño gigante, pero que desde que un paleontólogo estadounidense quiso llevarse una «tortuga» (Gliptodonte) de la pampa (finales de los años ochenta del siglo pasado), los coipaseños han ido mencionando cada vez más la presencia de estos animales en su territorio.

26 Cuando le pregunto a don Guillermo por este próximo diluvio, me introduce su respuesta con una larga narración sobre una vez que siendo niño enfermó de «susto» por haberse cruzado en el cerro con un ánima. La conclusión a la que llegó fue que igual que entonces, siendo católico, se pudo enfermar debido a la presencia del diablo, hoy en día no le sucedería por ser creyente. Porque Dios tiene presente quiénes son y quiénes no son creyentes, para cuando llegue este próximo diluvio de «fuego y azufre» salvar a los hermanos. 


\section{Conclusiones}

Recuperamos la teoría de Jean Pierre Bastian según la cual «los protestantismos durante este siglo y medio de desarrollo (...) se han latinoamericanizado con el pentecostalismo, al punto de asimilarse a la cultura de la región» (Bastian 2006: 53). Este autor realiza esta afirmación en contra de aquellos que piensan que es lo americano lo que se está pentecostalizando.

Existe, en ambas posiciones, un intento de entender por qué el sistema de creencias pentecostales o protestantes ha adquirido tanta fuerza en América, especialmente entre los sectores del denominado «catolicismo popular». Podríamos decir que desde una posición postestructuralista, las muchas etnografías que hoy se ocupan de dar una respuesta a esta cuestión, afirman que los pueblos americanos aceptan (las modernas) propuestas protestantes porque consiguen apropiarse de ellas y hacerlas comprensibles con códigos locales y conocidos. Estas etnografías demuestran

«que las creencias pentecostales hacen posible la libre expresión del mundo religioso popular habitado por demonios, espíritus, revelaciones y curas divinas. De tal manera que los creyentes reconocen en el pentecostalismo su religión, con profundas raíces en la cultura popular» (Bastian 2006: 50).

Lo que parece evidente es que, reconociendo el valor del sistema tradicional de creencias, éste aparece siempre en una posición de subordinación frente a las interpretaciones bíblicas. Estos análisis nos dicen que la conversión no es aceptada para reforzar el conocimiento tradicional, sino que más bien es éste el que permite que la conversión adquiera el suficiente valor y sentido para hacerse un lugar en el mundo americano. Es lo que sucede con la insistencia en resaltar la estrecha relación entre «la 'escatología' del mundo cíclico y el 'mesianismo'» con el mito del Inkarrí u otras profecías amerindias. Bastian lo explica muy bien:

«La asimilación entonces de estos protestantismos por la cultura religiosa y política de los milenarismos y los mesianismos, permite que los percibamos hoy en día más bien en continuidad que en ruptura con el universo cultural y religioso latinoamericano» (Bastian 2006: 51).

Ahora bien, escuchando las palabras de los evangélicos coipaseños que, repito, parecen tener toda la libertad interpretativa que su insularidad les permite, creo entender que el objetivo no es únicamente utilizar el conocimiento tradicional sobre el «tiempo del diluvio» para forzar una aceptación de las enseñanzas del Génesis, tanto en su comunidad de creyentes, como para el resto de la comunidad. Tampoco creo que su objetivo sea poner a los acontecimientos bíblicos al servicio del conocimiento tradicional, sino más bien encontrar el equilibrio adecuado entre el sistema comunitario de creencias y la fidelidad que deben guardar a las Sagradas Escrituras.

El corpus religioso protestante parece ser matizado en aras de un mejor reacomodo social, con el objetivo, y éste aspecto es el que quiero resaltar en estas conclusiones, de no provocar una ruptura total con la comunidad y, por ende, con un discurso ideológico y un sistema de creencias que ha sido parte siempre del imaginario de los creyentes.

Veíamos más arriba que Bastian afirmaba que la presencia de demonios y espíritus en el mundo amerindio era un terreno abonado para la creencia protestante, en este 
caso, de la presencia del diablo y de su mal en este mundo. Entre los hermanos coipaseños tenemos un ejemplo muy evidente.

Ante la presencia continua en la comunidad de enfermedades y males adjudicados a las chullpas (restos de esa antigua humanidad) los creyentes contestan siempre que «no creen» en ello. Esta falta de creencia no se refiere a su existencia (tanto la de los males, como la de las diferentes entidades que los puedan causar, así como de los estados ontológicos donde se las imaginan), de la cual no parecen tener duda, si no al hecho de que a ellos no pueda afectarles. Los hermanos están a salvo porque están bajo la protección del señor. Pero las chullpas existen, sus consecuencias malignas también. Pero también, como he mostrado en este trabajo, el pasado y la génesis que la comunidad les adjudica.

Los evangélicos parecen estar empeñados en encontrar un equilibrio entre ambos tipos de conocimiento, en que ninguno de ellos entre en contradicción con el otro, por medio de lo que creo es un rico ejercicio de interpretación local de la Biblia. El discurso evangélico coipaseño tiene como principal objetivo insertarse, como sector diferenciado, dentro del sistema tradicional y mayoritario de creencias por medio del conocimiento sobre el pasado comunario.

Parece más o menos evidente que esta «versión particular» del sistema religioso general, el protestante, «constituye una estructura autónoma de significación, que está construida, eso sí, con elementos (míticos y rituales) procedentes del sistema religioso canónico» (Gutiérrez 1984: 157). La propuesta aquí planteada nos puede ayudar, al mismo tiempo, a entender cómo se configura hoy el proceso religioso amerindio.

«[El] acudir a Dios o al demonio, a los nuevos y a los viejos dioses, a los santos y a los 'apus', a Jesucristo y al sol, configura el perfil de numerosos sistemas religiosos americanos y, aparentemente, resulta característico de los mismos y los diferencia de otros sistemas religiosos» (Gutiérrez 1984: 153).

No creo que sea éste el lugar y tampoco tal vez el debate epistemológico actual para discutir sobre el sincretismo o hibridación del sistema de creencia, y más concretamente del protestante, amerindio. Pero tal vez sí sea un primer paso para concederle una mayor importancia a la capacidad interpretativa de este sector de la población, capaz de crear un sistema religioso acorde con esta característica que siempre se ha atribuido a las poblaciones indígenas del continente: la de aceptar, incorporar y manejar ideas y elementos de la modernidad sin necesidad de romper de una forma total con las tradicionales.

\section{Referencias bibliográficas}

Abercrombie, Thomas A.

1998 Pathways of Memory and Power. Ethnography and History among an Andean People. Madison: The University of Wisconsin Press.

Allen, Catherine J.

1994 «Time, Place and Narrative in an Andean Community». Bulletin de la Société Suisse des Américanistes 57-58: 89-95. 
ANDRADE, Susana

2004 Protestantismo indígena. Procesos de conversión religiosa en la provincia de Chimborazo. Quito: Abya-Yala - FLACSO - IFEA.

2005 «Iglesias evangélicas y pentecostales quichuas en la provincia de Chimborazo, Ecuador», en De indio a hermano: Pentecostalismo indígena en América Latina, Bernardo Guerrero et al, eds., pp. 77-110. Iquique: Ediciones Campvs.

BASTIAN, Jean Pierre

2006 «De los protestantismo históricos a los pentecostalismos latino-americanos: análisis de una mutación religiosa». Revista de Ciencias Sociales 16: 38-54.

CANESSA, Andrew

2000 «Contesting Hybridity: Evangélicos and Kataristas in Highland Bolivia». Journal of Latin American Studies 32 (1): 115-144.

2012 Intimate Indigeneities. Race, Sex, and History in the Small Spaces of Andean Life. Durham: Duke University Press.

CASAVERde Rojas, Juvenal

1970 «El mundo sobrenatural en una comunidad». Allpanchis Phuturinqa 2: 121-243.

Choque Canqui, Roberto y Cristina Quisbert QuisPe

2006 Educación indigenal en Bolivia. Un siglo de ensayos educativos y resistencias patronales. La Paz: Unidad de Investigaciones Históricas - Ibis.

Cruz, Pablo

2006 «Mundos permeables y espacios peligrosos. Consideraciones acerca de punkus y qaqas en el paisaje altoandino de Potosí, Bolivia». Boletín del Museo Chileno de Arte Precolombino 11 (2): 35-50.

Fernández JuÁrez, Gerardo y Xavier Albó Corrons

2008 «Pachjiri. Cerro sagrado del Titicaca». Revista Española de Antropología Americana 38 (1): 239-255.

Gil García, Francisco M.

2007 «Las ruinas, la iglesia, la mina. Identidad local y construcción del discurso histórico de una comunidad del Altiplano de Lípez (Dpto. Potosí, Bolivia)», en El Mediterráneo y América, J. J. Sánchez Baena y L. Provencio Garrigos, comps., pp. 663-676. Murcia: Editora Regional de Murcia.

GuTIÉRrez Estévez, Manuel

1984 «En torno al estudio comparativo de la pluralidad católica». Revista Española de Investigaciones Sociológicas 27: 137-174.

La Santa Biblia

2005 La Santa Biblia. Antiguo y Nuevo Testamento. Antigua versión de Casiodoro de Reina (1569) revisada por Cipriano de Valera (1602). Otras revisiones: 1862, 1909 y 1960. Londres: Bibles.org.uk.

LaLive D'Epinay, Christian

1968 El refugio de las masas. Estudio sociológico del protestantismo chileno. Santiago de Chile: Editorial del Pacífico.

LAPORTA, Héctor

2005 «La religión evangélica en la región andina», en Enciclopedia iberoamericana de religiones. Volumen 4: Religiones andinas, Manuel M. Marzal, ed., pp. 287-309. Madrid: Trotta. 
Marzal, Manuel M.

1971 El mundo religioso de Urcos. Un estudio de antropología religiosa. Cuzco: Instituto de Pastoral Andina.

2002 Tierra encantada. Tratado de antropología religiosa de América Latina. Madrid: Pontificia Universidad Católica del Perú - Trotta.

MÉTRAuX, Alfred

1931 «Un mundo perdido. La tribu de los chipayas de carangas». Sur 1: 98-131.

Molinié, Antoinette

2005 «La transfiguración eucarística de un glaciar: una construcción andina del Corpus Christi», en Etnografías del Cuzco, A. Molinié, comp., pp. 69-88. Lima: Centro de Estudios Regionales Andinos «Bartolomé de Las Casas» - Instituto Francés de Estudios Andinos.

Muñoz MorÁn, Óscar

s.f. «'Ya no hay pasantes'. La fiesta patronal y la descomposición de un ayllu quechua». Comunicación. International Congress of Americanists. Viena, Austria, junio 2012.

Nicolas, Vicent, Sandra Zegarra y Alfredo Puma (recops.)

2004 Ayllusninchismanta parlarispa. Antología de historias orales de Tinkipaya. La Paz: PIEB.

Nuñez del Prado BÉJAR, Juan V.

1970 «El mundo sobrenatural de los quechuas del sur del Perú a través de la comunidad de Qotobamba». Allpanchis Phuturinqa 2: 57-119.

RASNAKE, Roger

1988 «Images of Resistance to Colonial Domination», en Rethinking History and Myth. Indigenous South American Perspectives on the Past, Jonathan D. Hill, ed., pp. 136-156. Chicago: University of Illinois Press.

RicARd Lanata, Xavier

2007 Ladrones de sombra. El universo religioso de los pastores del Ausangate. Lima: Instituto Francés de Estudios Andinos - Centro de Estudios Regionales «Bartolomé de las Casas».

RIVIÈRE, Gilles

2007 «Bolivia: el pentecostalismo en la sociedad aimara del Altiplano». Nuevo Mundo Mundos Nuevos. Documento electrónico, <http://nuevomundo.revues.org/6661>, con acceso el 4/2/2014.

SENDÓn, Pablo F.

2010 «Los límites de la humanidad. El mito de los ch’ullpa en Marcapata (Quispicanchi), Perú». Journal de la Société des Américanistes 96 (2): 133-179.

Strobele Gregor, Juliana

1989 Indios de piel blanca: evangélicos fundamentalistas en Chuquiyawu. La Paz: Hisbol.

TolA, Florencia C.

2011 «'Todos los hombres nacieron en Jerusalem'. Mito y Evangelio en las narraciones sobre los orígenes de la humanidad entre los toba (qom) del Chaco argentino». Anthropologica 29: 167-186. 
Torre López, Arturo Enrique de la

2005 «'La más rigurosa secta de nuestra religión'. La Asociación Evangélica de la Misión Israelita del Nuevo Pacto Universal», en Enciclopedia iberoamericana de religiones. Volumen 4: Religiones andinas, Manuel M. Marzal, ed., pp. 311-357. Madrid: Trotta.

WACHTEL, Nathan

2001 El regreso de los antepasados. Los indios urus de Bolivia, del siglo XX al XVI. México: El Colegio de México - Fideicomiso Historia de las Américas - Fondo de Cultura Económica. 